\title{
Image Terminal Series of Hokkaido University Picture Archiving Communication System
}

\author{
Yusuke Ozaki and Kazuo Miyasaka
}

\begin{abstract}
In Hokkaido University Hospital, the large-scale PACS has been in clinical use since 1989. We developed various kinds of imaging terminals such as the image input terminal and the image display terminal for this system. This article presents detailed description of these terminals.
\end{abstract}

Copyright $(1991$ by W.B. Saunders Company

$\mathbf{T}$ HE TERMINALS of the Hokkaido University Hospital picture archiving and communication system (HU-PACS) can be divided into two types: terminals for image input and terminals for image display (Fig 1). The image input terminal group accomplishes the collection of images as digital data. This group is further divided into film input terminals (FIT) that can digitize images from $\mathrm{x}$-ray films and digital image input terminals (DIT) that act to acquire on-line digital images from computed tomography (CT), magnetic resonance imaging (MRI), and computed radiography (FCR). IDT retrieve images from a host computer and display them on a cathode-ray tube (CRT) monitor for diagnosis or review and thus play a most important role among the units of PACS. They include two kinds: image work station (IWS) that are installed in the radiology department and IDT that are distributed to some outpatient clinics. We provide detailed description of the individual components of the terminals.

\section{FIT AND DIT}

HU-PACS has 10 image input terminals, including 1 FIT and 9 DITs that receive digital images from various modalities. Älthough all input systems related with digital data are equally included in the category of DIT, the interfaces vary according to modalities, which inevitably causes differences not only in hardware composition but also in transfer rate, communication protocol, and image display formatting, depending on modalities.

\section{IDT}

IDTs are distributed to several outpatient clinics in the hospital and are characterized by the following features: (1) They are designed to occupy less room, (2) they allow easy operation through a mouse so that anyone can handle them without difficulty, (3) each is equipped with a nonglare-coated, noninterlaced cathode-ray tube (CRT) monitor so that there is less reflection and flickering, thereby causing less visual fatigue, (4) they are capable of multiple functions such as display of a retrieval list and simultaneous display of two images, and (5) each uses a dedicated image processor capable of high-speed retrieving and processing.

\section{System Configuration}

The hardware composition of an IDT is shown in Fig 2. Image data transferred from a host computer are compressed to one-third to one-tenth of the original amount of data so that a 80 Mbyte magnetic disk can store about 150 sheets of images. For display, those images are expanded to their original size by a compression/expansion processor.

An IDT has hardware specialized for individual functions such as edge enhancement and magnification so that image processing can be managed rapidly. The control processor which controls the IDT can simultaneously receive images from a host computer and manipulate the image.

\section{Function}

Retrieval. To increase speed and moderate load in a local area network (LAN), the IDT is specially designed to transfer image data automatically to the terminal's magnetic disk without deliberate commands from the operator.

Because an ordering terminal is always placed close to an IDT in HU-PACS, reference images stored in the host as well as images obtained on a given day are transferred automatically from the host computer to the terminal disk. This is accomplished in the order of appointment by commands from the operator.

Another mode is also available: an operator who wishes to see a series of images can call them back from the host computer and, while viewing

From the Medical Equipment Division, NEC Corporation, and the Department of Radiology, Hokkaido University Hospital. Hokkaido, Japan.

Address reprint requests to Yusuke Ozaki, Medical Equipment Division.

Copyright (C) 1991 by W.B. Saunders Company

0897-1889/91/0404-0107\$03.00/0 


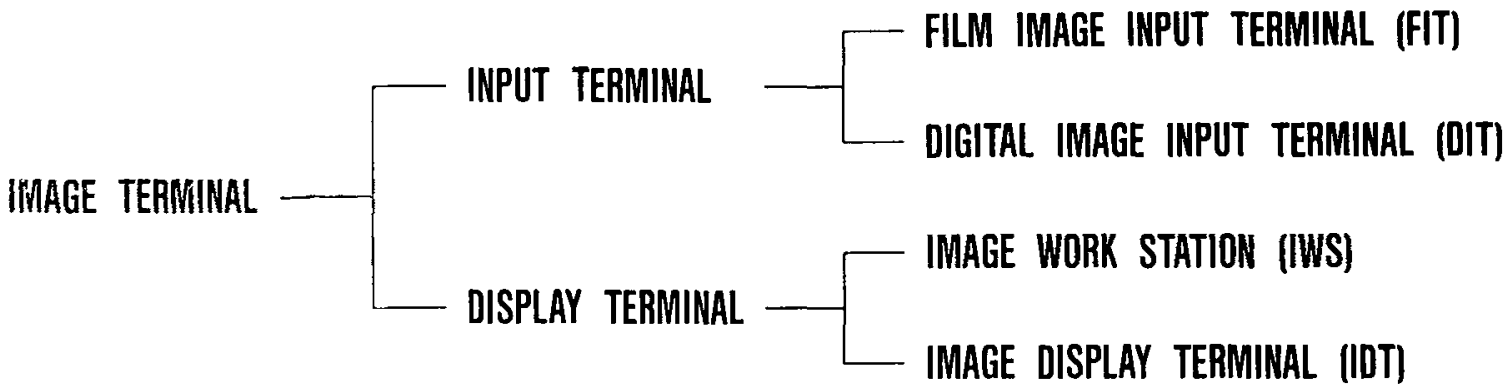

Fig 1. Image terminal series of HU-PACS.

the first image on CRT, have the images automatically transferred in order to the magnetic disk to be served immediately for viewing.

Retrieval can be accomplished simply by operating a mouse if the relevant data are stored in the magnetic disk in the terminal. In this case, the retrieval unit is limited to one examination normally. An operator who wishes to make conditional retrieval can enter necessary data such as modality, region of examination, date, and ordering department through the keyboards and send the order to the image control processor by clicking the mouse.

Image display. An IDT, although equipped with 1 CRT, has a function that provides two different displays as though it had 2 CRTS by splitting the screen into right and left halves. The display matrix of the CRT is 1024 (in height) $\times 768$ (in width) $\times 2$. Because most images are oblong (except for $14^{\prime} \times 14^{\prime}$ films), an image displayed on the split screen is virtually the same in terms of image quality as the full-sized one.

For retrieving images, a high-resolution mode and a standard mode are available. In the highresolution mode, an image is stored with a maximum matrix of $2048 \times 2048$. A portion may be displayed to fit the display matrix, but shows the full resolution. This is especially apparent when the display is magnified.

In standard mode, an image is stored with the matrix equal to that in the display; its resolution remains the same. However, in this mode, the next image is automatically read from magnetic disk into the vacant memory area in advance, so that it is able to minimize image switching time. This mode is especially useful for viewing a series of images taken a particular day.

Image processing. The image processing menu consists of three modes: two independent modes and one mode for selecting appropriate commands for image processing. The former includes density windowing and image magnifying/ scrolling. These three modes can be selected in a cyclic manner by mouse click, and for operators who are not accustomed to using a mouse an icon is also provided at one corner of the CRT.

An IDT has hardware dedicated for every function necessary for image processing, which allows speedy manipulation of image data. Thus, edge enhancing can be performed as rapidly as retrieving from $\mathrm{HD}$, and local areas can be magnified with 1 to 256 steps of data interpolation simultaneously with the movement of handling a mouse.

Appropriateness of gamma-curve correction is important when dealing with images provided through various modes for display. We have been engaged in finding an appropriate algorithm by which the control processor can automatically determine the gamma-curve correction for a given imaging condition, including part and method of examination, but have not yet been successful. At present, we manage to provide every department with a number of gamma curves that have been frequently used there.

In addition, a department often requires some functions of image manipulation; eg, an orthopedic department requires measurement of an angle between two independent lines or the distance between them. Considering that in the future image processing will be expanded to other medical departments, it is important to provide IDT with sufficient flexibility to meet a wide variety of requirements.

\section{IWS}

An IWS is installed at the center in the radiology department where it serves as a terminal through which radiologists read images. Thus, it 


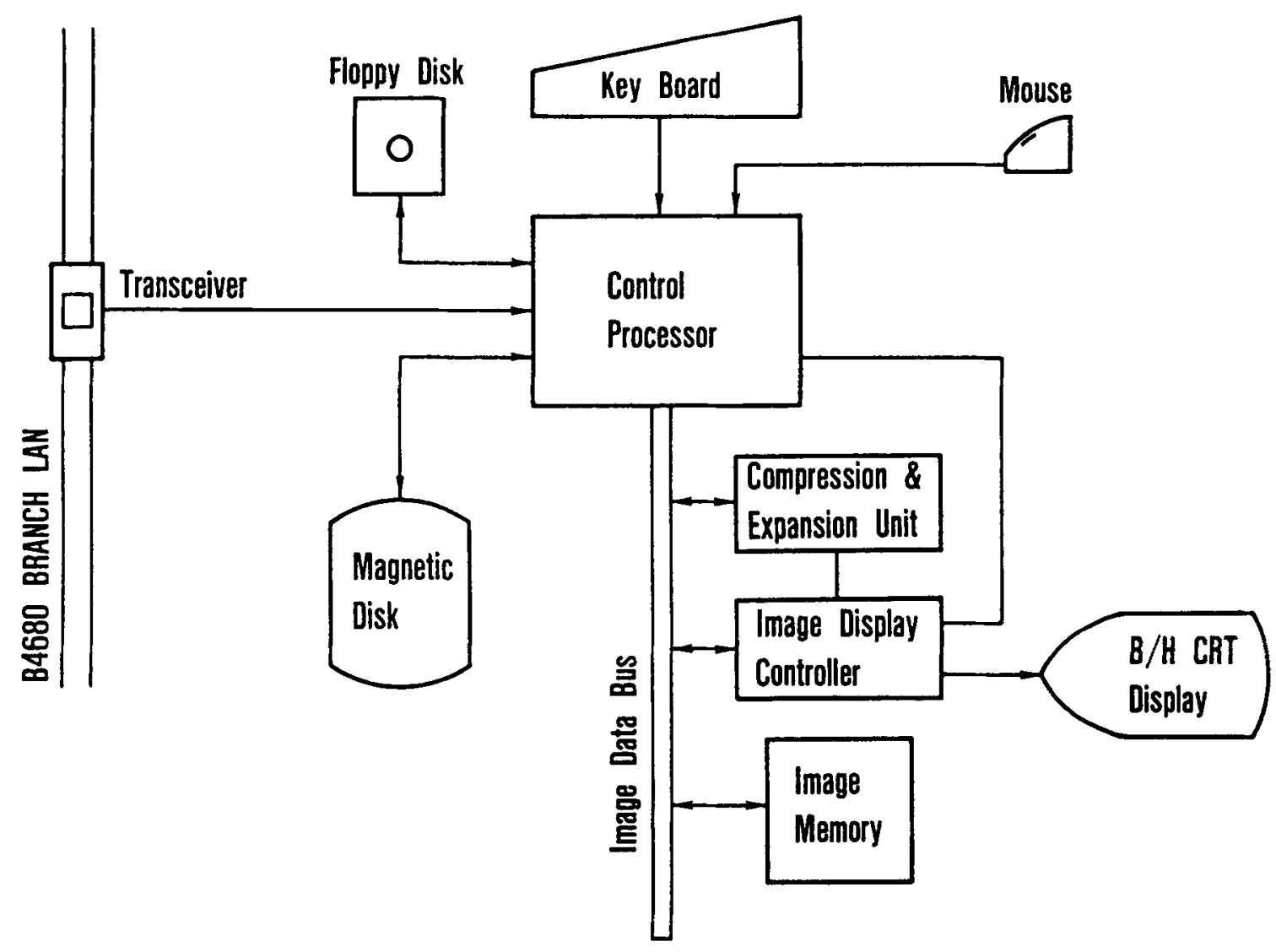

Fig 2. Image display terminal block diagram.

has concept slightly different from that of IDT (Fig 3).

IWS has three CRTs: two for image display and one for character display. They are assigned different functions. The two CRTs can display two different images at the same time with an independent memory of 16 Mbytes for each CRT, thereby enabling more complex processing. Its magnetic disk also has a larger memory (160 Mbytes) that can store a series of images.

\section{System Composition}

The IWS is controlled with the control processor, and image processing is achieved by the image processing unit. In contrast to IDT which has a hardware dedicated for every image processing, the image processing unit of IWS is a programmable unit utilizing an assembler specialized for the purpose.

Image memory has a capacity of 32 Mbytes in all. Two sets of an original image ( 8 Mbytes) and its processed image ( 8 Mbytes) are stored in image memory. For a special purpose such as calcula- tion between two images, however, dynamic addressing of the memory is possible.

\section{Function}

Retrieval and display. The function of the retrieval key and its method is fundamentally the same as in IDT except that in IWS a retrieval list is always displayed on the screen because the CRT for dialogue is available as an independent unit in IWS. For images stored in the disk at the operator's terminal, most processing can be achieved solely by mouse operation. Thus, to obtain the menu for image processing, one need only click a mouse at a desired point where the menu unfolds.

PACS images registered in the host computer can be retrieved and read freely from a terminal in a department, but to limit this function to a certain degree a function called permission is added. For example, if some images are taken in the radiology department, they are marked for a certain initial period with permission flags that protect the images from access by other terminals. 


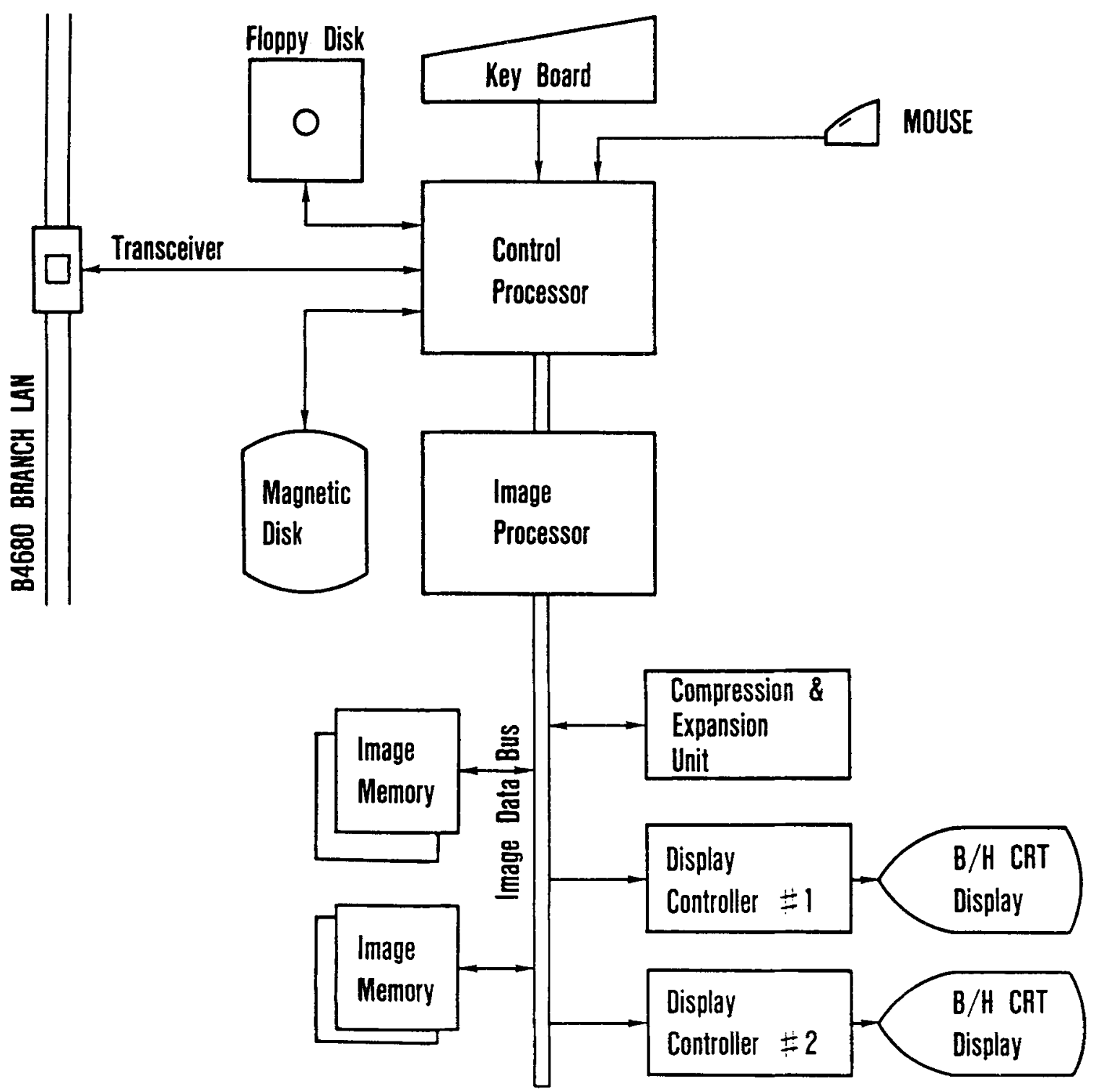

Fig 3. Image workstation block diagram.

When the radiologist makes a report on diagnosis of those images, the flags are reset so that access to the images from other departments becomes possible.

Image processing. One of the most effective functions of digital image processing is mathematical manipulation of different images. As a typical example, a subtraction function allows enhancement of an area invaded with a contrast medium; the image data taken after medium injection is subtracted by the comparable image before injection.

Alternatively, when two image data are overlapped, the resultant image looks as though the same images on film were overlapped. There are, considerable differences between the two types of image overlapping, however: In digital image processing either image can be moved in a parallel direction simply by moving a mouse and overlapping films never fail to darken the resultant image, whereas in computer-controlled image overlapping brightness of the overlapped image on CRT can be adjusted to an appropriate level by using density window processing.

Images obtained through CT or MRI are fed to DIT or an input terminal where they are contracted to nine images per frame and registered as such in the host computer. Thus, when a re- 
trieval is made through IWS, each frame of 9 slices constituting a part of one examination is displayed simultaneously. This procedure is adopted mainly because it allows faster data transfer and display and partly because the images originally have fewer pixels and it has been customary to place radiological images side by side for easy comparison.

IWS allows the panel to be divided into two or more elements so that for CT images a maximum of 72 slices can be viewed at one time. IWS also has functions useful for data analysis: a plotting function by which to transform data on a given line into a graph and a function to construct a histogram from data within the region of interest (ROI) supplemented with histogram homogenizing performance.

\section{CONCLUSIONS}

PACS aims at ease of use so simple and speedy that even physicians long accustomed to previous diagnostic methods can freely exploit it for their clinical needs using CRT and keyboard instead of $\mathrm{x}$-ray films and light boxes. Smooth introduction of this system was possible mainly because it presents two operation systems: icon mode for the novice and mouse-click mode for the expert for whom quick command input is essential. 